\title{
A SINGLE INSTITUTION EXPERIENCE IN HYPERTROFIC PYLORIC STENOSIS
}

\author{
TAMAS TOTH ${ }^{1}$, ANA-MARIA PRIȘCA ${ }^{2}$, ANGELA BORDA ${ }^{3}$, HOREA GOZAR $^{4}$, \\ RADU-ALEXANDRU PRIȘC $\check{A}^{5}$ \\ ${ }^{1,4,5}$ County Emergency Clinical Hospital of Târgu Mureș, ${ }^{2}$ Integrative Medicine Center "Lotus Life”, Târgu Mureș, \\ 3 "George Emil Palade” University of Medicine, Pharmacy, Science and Technology of Târgu Mures,
}

\begin{abstract}
Keywords: $\quad$ hypertrofic Abstract: The most common causes of non-bilious vomiting in infants are hypertrophic pyloric pyloric stenosis, non- stenosis and pylorospasm. Differential diagnosis between the pathologies establishes the correct bilious vomiting, treatment. The aim of our study is to present the management of the pathologies cured in our ultrasonography department. The longer the duration of vomiting symptoms the higher muscle thickness was shown. Dehydration and lethargy was associated with higher muscle thickness. There were no intraoperative or postoperative complications recorded in our cases. Ultrasonography is the gold standard in imaging technique but it requires an experienced radiologist. In case of negative physical examination and ultrasonography, but high suspicion of the diagnosis a fluoroscopy may be required. The correct diagnosis avoids unnecessary surgery combined with general anesthesia.
\end{abstract}

\section{INTRODUCTION}

Hypertrophic pyloric stenosis and pylorospasm are the most frequent causes of nonbilious vomiting in children during the first 3 months beside of idiopathic gastroesophageal reflux.(1)

Pylorospasm is defined by the lack of coordinated gastric emptying. Normally, the gastric emptying is coordinated by sequential contractions from the antrum to the pyloric region and duodenum. In this condition the gastric emptying is delayed, the antral peristalsis is functioning and the pylorus is elongated.(2)

Hypertrophic pyloric stenosis with an incidence of 4 per 1000 live births in Western Countries is commonly presented in male infants during the first 2 to 12 weeks of life. The most frequent symptom is repeated, non-bilious vomiting after feeding which is initially non-projectile that with time progresses to a projectile one. Persistent vomiting leads to lethargy and dehydration with hyper or hyponatremia and hypochloremic metabolic alkalosis. Diagnosis can be established easily if an olive-shape, firm pyloric muscle mass can be palpated.

Differential diagnosis is important between hypertrophic pyloric stenosis and pylorospasm because of the treatment options. The treatment of hypertrophic pyloric stenosis is pyloromyotomy and for pylorospasm is conservative by observation and antispasmodics.

Abdominal ultrasound has replaced barium studies in establishing the diagnosis and had become the diagnostic method of choice. The guidelines consider as pathologic limits 3 $\mathrm{mm}$ for pyloric muscle thickness and $15 \mathrm{~mm}$ for pyloric length. Rohrschneider et al. (3) considered the muscle thickness the most discriminating factor. Infants with equivocal physical examination and ultrasound should undergo to barium upper gastrointestinal study in order to establish the correct diagnosis.(4)

The aim of our study is to describe the clinical characteristics of patients with infantile hypertrophic stenosis, management and its outcome in the Department of Pediatric Surgery and Orthopedics of County Emergency Clinical Hospital of Târgu Mureș.

\section{MATERIALS AND METHODS}

Our retrospective study comprised 24 infants aged between 0 and 2 months who had been presented with suspected hypertrophic pyloric stenosis at the Department of Pediatric Surgery and Orthopedics, County Emergency Clinical Hospital of Târgu Mureș between 2014 and 2020. The collected data were patients' demography categorized by sex and age, clinical presentation, duration of symptoms and ultrasound or barium upper gastrointestinal study findings.

Ultrasonographic criteria for hypertrophic pyloric stenosis is represented by a muscle thickness more than $3 \mathrm{~mm}$. Fluoroscopy was done in cases where the physical examination and ultrasound findings were equivocal. The final diagnosis was made after correlating the clinical, laboratory and ultrasonographic or barium upper gastrointestinal study findings.

\section{RESULTS}

24 infants were admitted to our Hospital with suspected hypertrophic pyloric stenosis. $87.5 \%$ of them were male infants and $83.3 \%(n=20)$ were full-term. $58.3 \%$ presented non-bilious vomiting for more than a week before admission, $70.83 \%$ was dehydrated in some degree and $62.5 \%$ presented lethargy. Laboratory data showed hypocloremic metabolic

${ }^{2}$ Corresponding author: Ana-Maria Prişcă, Str. Gh. Marinescu, Nr. 50, Târgu Mureş, România, E-mail: anamariacodreanu@gmail.com, Phone: $+40757401776$

Article received on 07.07.2020 and accepted for publication on 02.12.2020 


\section{CLINICAL ASPECTS}

alkalosis in $50 \%$ of the infants who presented vomiting more than a week. The olive-shaped pyloric muscle mass was palpated in $45.83 \%$ of the cases. 21 infants $(87.5 \%)$ had the positive ultrasonographic criteria for hypetrophic pyloric stenosis; the mean muscle thickness was $4.92 \pm 1.62 \mathrm{~mm}$.

A statistically significant difference was found between the duration of vomiting and muscle thickness $(\mathrm{p}<0.01)$. The longer the duration of vomiting symptoms the higher muscle thickness was shown. Muscle thickness was higher in the group where the olive mass was palpable but without any statistical significance. Dehydration and lethargy was associated with higher muscle thickness $(\mathrm{p}=00.1, \mathrm{p}=00.2)$.

In 3 cases the physical examination and abdominal ultrasound was equivocal. The infants were vomiting for less than a week, two of them with symptoms of dehydration. The mean muscle thickness in these cases was $2.7 \mathrm{~mm}$. The fluoroscopy showed delayed gastric emptying. These infants were treated conservatively by observation and antispasmodics. The evolution of these patients was satisfactory; they were discharged from the hospital after 6-7 days.

\section{DISCUSSIONS}

Hypertrophic pyloric stenosis is characterized by hyperplasia of smooth muscle that leads to pyloric canal narrowing and gastric outlet obstruction. Male infants were affected in our study in 6.6:1 ratio comparable with data from the literature of 4-6:1 ratio.(5)

The most frequent presentation symptom was nonbilious vomiting. Persistent vomiting can lead to dehydration, weight loss, and lethargy and hypocloremic metabolic alkalosis. The olive shape mess palpable in the epigastric region was present in $45.83 \%$ of the cases compared to Macdessi J et al (6) work who states that clinical diagnosis of pyloric stenosis is a declining art. Clinical and laboratory findings can be related to the duration of the symptoms. Hamdi Hamed A (7) found a statistically significant correlation between the muscle thickness and duration of vomiting, presence of dehydration and lethargy which is explained with the pathophysiological mechanism of the illness. Our study showed the same findings.

Muscle thickness is higher among lethargic, dehydrated infants whom are vomiting longer than a week. Starinsky et al. (8) described a muscle thickness increment after effective fluid resuscitation. Diagnosis is hard to establish based only on clinical symptoms. It is important to differentiate the pathology which needs surgery (hypertrofic pyloric stenosis) and conservative treatment (pylorospasm) (figure no. 1.) After pyloromyotomy the muscle thickness gradually returns to normal.

Figure no. 1. Longitudinal oblique plane. A. Cervix sign in HPS - elongated and thickened pylorus resembling a cervix. B. Fluid present in the antrum, normal muscle thickness $(2.5$ mm) suggestive for pylorospasm

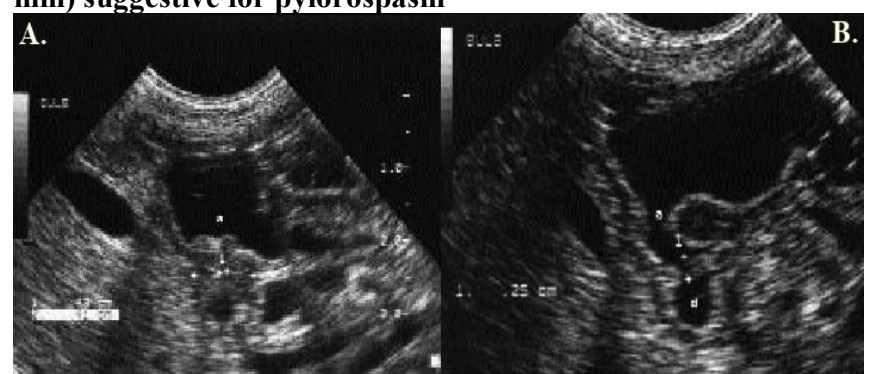

Diagnostic imaging is useful in equivocal physical examination. Ultrasound is the diagnostic method of choice with a sensitivity ranging from 76 to $100 \%$ and specificity of 85 to $100 \% .(9)$
The most common measurement criteria for hypertrofic pyloric stenosis is a pyloric muscle thickness greater than $3 \mathrm{~mm}$, a transverse pyloric diameter greater than 13 mm.(10)

Other signs suggestive for pyloric stenosis are described by radiologists as target sign, cervix sign and antral nipple sign. However ultrasound is a non-invasive and nonirradiative, cheap dynamic imaging study, it is hard to examine a newborn. A stomach filled with gas or milk can mislead the diagnosis by displacing the pylorus posterior.(11) In preterm infants the smaller weight and size should be taken into consideration. Argyropoulou et al (12) demonstrated that pyloric dimensions are increasing with the gestational age and body weight.

In our study we performed fluoroscopy in 3 cases because of equivocal clinical examination and borderline measurements. Positive findings would have been an elongated pyloric canal, active gastric hyperperistaltis (caterpillar sign), and double or triple track sign. Delayed gastric emptying was present which put the diagnosis of pylorospasm. The conservative treatment was effective and the patients were discharged after 6-7 days.

All of the patients with the diagnosis of hypertrophic pyloric stenosis underwent surgery (Fredet-Ramstedt extramucosal pylorotomy). In the literature there is mortality less than $1 \%$ (13) which occurs from fluid and electrolyte imbalance. An intraoperative complication such as mucosal perforation is described in $1-2 \%$ (14) of the cases. We had no intraoperative or postoperative complications.

\section{CONCLUSIONS}

The triad of visible gastric peristalsis, non-bilious projectile vomiting and palpable epigastric olive mass is not always present.

Physical examination can be equivocal where diagnostic imaging can help. Abdominal ultrasound is the first choice but in case of negativity and high suspicion of the diagnosis a barium upper gastrointestinal study should be performed.

Ultrasonography measurements require an experienced sonographer. The diagnosis should be established correlating the clinical and imaging data in order to avoid the unnecessary surgical treatment.

\section{REFERENCES}

1. Panteli C, New insights into the pathogenesis of infantile pyloric stenosis. Pediatr Surg Int. 2009;25:1043-1052.

2. Anthony G, Jared D, Harris L.C. Pylorospasm (Simulating Hypertrophic Pyloric stenosis) with secondary Gastroesophagal Reflux. Ultrasound Quarterly. 2008;24(2):93-96.

3. Rohrschneider WK, Mittnacht H, Darge K, Troger J. Pyloric muscle in asymptomatic infants: sonographic evaluation and discrimination from idiopathic hypertrophic pyloric stenosis. Pediatr Radiol. 1998;28(6):429-34.

4. Marija F, Marina SK, Zeljka P, Vinka BB, et al. Diagnosting imaging of hypetrophic pyloric stenosis (HPS). Radio Oncol. 2001;35(1):11-16.

5. Korgh C, Gortz S, Wohlfahrt J, Biggar RJ, Melbye M, Fischer Tk. Pre- and perinatal risk factors for pyloric stenosis and their influence on the male predominance. Am J Epidemiol. 2012;176(1):24-31.

6. Macdessi J, Oates RK. Clinical diagnosis of pyloric stenosis: a declining art. BMJ. 1993;306:553-555.

7. Hamdi HA. Is there a relation between pyloric muscle thickness and clinical and laboratory data in infants with hypetrophic pyloric stenosis? Indian J Surg. 
2015;77(3):827-830

8. Starinsky R, Klin B, Siman TY et al Does dehydration affect thickness of the pyloric muscle? An experimental study. Ultrasound Med Biol. 2002;28:421-423.

9. Forster N, Haddad RL, Choroomi S, Dillet AV et al. Use of ultrasound in 187 infants with suspected infantile hypertrophic pyloric stenosis. Australas Radiol. 2007;54:560-563.

10. Reed AA, Michael K. Hypertrophic pyloric stenosis. J Diagn Med Sonography. 2010;26:157-160.

11. Silvia CD, Sophie S, Helena T. et al. Hypertrophic pyloric stenosis: tips and tricks for ultrasound diagnosis. Insights Imaging. 2012;3:247-250.

12. Argyropulou MI, Hadjigeorgi CG, Kiortsis DN. Antropyloric canal values from early prematurity to full term gestational age: an ultrasound study. Pediatr Radiol. 1998;28:933-936

13. Rene N, Paul NT, Faustin FMT et al. Infantile hypertrophic pyloric stenosis: A 4-year experience from two tertiary care centres in Cameroon. BMC Res Notes. 2008;11:33.

14. Taqi E, Boutros J, Emil S et al. Evaluation of surgical approaches to pyloromyotomy: a single-center experience. J Pediatr Surg. 2007;42(5):865-868. 